\title{
The problems of fishermen in the Southern Aral Sea region
}

\author{
Bakhtiyor Karimov $\cdot$ Helmut Lieth $\cdot$ Mohira Kurambaeva · Irina Matsapaeva
}

Published online: 12 June 2007

(C) Springer Science+Business Media B.V. 2007

Erratum to: Mitig Adapt Strat Glob Change 10(1):87-103

DOI 10.1007/s11027-005-7832-0

This Erratum has been initated inorder to add further corrections. The new corrections are:

page 87: $3000 \mathrm{mg} \mathrm{yr}^{-1}$ should be 3.000 tons $/ y r$ and $6000 \mathrm{mg} \mathrm{yr}^{-1}$ should be 6.000 tons $/ y r$ page 88: Kazakhstan $(\times 103 \mathrm{mg})$ should be Uzbekistan, tons

page 90: $550-20 \times 103 \mathrm{ha}^{-1}$ should be $h a$

page 92: $32 \mathrm{mg} \mathrm{yr}^{-1}$ should be 32 tons $/ y r$, and $1.000 \mathrm{Mg}$ should be 1.000 tons

page 94: $17 \mathrm{Mg}$ should be 17 tons

page 99: $552 \mathrm{Mg}$ should be 552 tons

page 101: $459 \mathrm{Mg}$ should be 459 tons, $1.500 \mathrm{Mg}$ should be 1.500 tons, $552 \mathrm{Mg}$ should be 552 tons, $3000 \mathrm{Mg}$ should be 3.000 tons

The online version of the original article can be found under doi: 10.1007/s11027-005-7832-0

B. Karimov · M. Kurambaeva · I. Matsapaeva

Institute of Water Problems of Uzbekistan Academy of Sciences, Tashkent, Uzbekistan

H. Lieth $(\bowtie)$

Institute for Environmental Systems Research, University of Osnabrueck, Osnabrueck, Germany e-mail: Helmut.lieth@usf.uni-osnabrueck.de 